MATHEMATICS OF COMPUTATION

Volume 73, Number 248, Pages 1601-1622

S 0025-5718(04)01645-X

Article electronically published on April 27, 2004

\title{
ALGEBRAIC ALGORITHMS FOR THE ANALYSIS OF MECHANICAL TRUSSES
}

\author{
I. BABUŠKA AND S. A. SAUTER
}

\begin{abstract}
Infinite periodic lattices can be used as models for analyzing and understanding various properties of mechanical truss constructions with periodic structures. For infinite lattices, the problems of connectivity and stability are nontrivial from the mathematical point of view and have not been addressed adequately in the literature. In this paper, we will present a set of algebraic algorithms, which are based on ideal theory, to solve such problems.

For the understanding of the notion "complicated three-dimensional lattices", it is essential to have this paper with colored figures.
\end{abstract}

\section{INTRODUCTION}

Lattice models are used in many applications such as models of heterogeneous materials ([16], [8]), fracture models ([17]), porous media ([7], [4]), and biophysics (10]). For a survey of some applications, we refer to [16] and [18]. Lattices are becoming more and more interesting for industrial production because these materials are light, cheap, and can be designed to prescribed stiffness requirements.

In this paper, we will investigate infinite periodic lattices which may serve as approximations to large periodic structures. Various aspects, such as the investigation of Green's function for lattice equations for (infinite) periodic lattices, are addressed in the literature (see, e.g., 9], [11, 14]). In 22, fast solution methods of equations on finite lattices will be presented.

Fourier analysis can be naturally applied to infinite periodic lattices. Questions of connectivity and stability (rigidity) are directly related to the problems of existence and uniqueness of the solutions of equations on infinite lattices. However, to decide whether an infinite lattice is connected and rigid is by no means trivial and we will develop algorithms for this purpose in this paper.

For vector problems, the stability is closely related to the rigidity of the skeletons and frameworks. This problem is a classical one in structural mechanics and methods for the verification of the rigidity of a lattice are presented in [5], 12], 11. The drawback of all these approaches is that they become very costly or even fail if the lattices contain very long connections or are even infinite. In our paper, we will develop algebraic algorithms which perform efficiently, especially in such cases.

We will consider scalar and vector problems on infinite periodic lattices. In a mathematical setting which will be introduced, the problems can be formulated in a variational way by a bilinear form acting on grid functions which are defined at the nodes of the lattice. Alternatively they can be expressed by finite difference

Received by the editor September 4, 2002 and, in revised form, March 19, 2003.

2000 Mathematics Subject Classification. Primary 65T50, 06B10, 35J55.

(C)2004 American Mathematical Society 
operators. Based on the periodicity of the lattice, we may associate with such finite difference operators a matrix-valued symbol. Because the symbol and the inverse of the symbol are related to the stability of the lattice equations and the continuous dependence of the solution on the right-hand side, the investigation of the zeroes of the determinant is equivalent to the analysis of the connectivity and stability. We

will present purely algebraic algorithms which decide whether the determinant of the symbol has a zero only at the origin or whether there exist further zeroes.

The algorithms which we are going to present have purely algebraic character in the sense that no numerical approximation is involved and they manipulate the data in the ring of vector-valued integers.

Although we will address only the lattice in the entire $\mathbb{R}^{d}$, the results together with the Fourier analysis approach can be used for analysis of local damages and for an approximate solution on finite lattices (see, e.g., [13], [14, [15], [6], [8]).

The paper is structured as follows.

In Section 2, we will formulate discrete vector potentials on lattices which have the same abstract form as problems in linear elasticity. We will discuss the problem of stability of these potentials in terms of the symbol of the underlying difference operator. We will see that the connectivity of the lattice and the structure of the set of zeroes of the determinant of the symbol play an essential role in the stability.

In Section 3. we will present a purely algebraic algorithm for deciding in finite time whether or not an infinite periodic lattice is connected.

In Section 4, an algebraic algorithm will be introduced for deciding in finite time whether or not the discrete vector potential equations are stable. We apply these algorithms to some characteristic model problems.

\section{Discrete Vector potentials on General LAtTices}

In this section, we will introduce an abstract mathematical framework for lattice equations. We begin by introducing the geometry and the graph of the lattices.

2.1. The geometry of lattices. In this subsection, we will introduce periodic lattices in $d$ dimensions.

Periodic lattices are most easily defined as periodic copies of points and edges of a reference cell. The reference cell is a bounded domain $\omega \subset \mathbb{R}^{d}$. To obtain a macro-periodic structure, we assume that, for given vectors $t^{(i)} \in \mathbb{R}^{d}, 1 \leq i \leq d$, the integer translates

$$
\omega_{m}:=\left\{y \in \mathbb{R}^{d} \mid \exists x \in \omega: y=x+\sum_{i=1}^{d} m_{i} t^{(i)}\right\} \quad \forall m \in \mathbb{Z}^{d}
$$

are disjoint and satisfy $\mathbb{R}^{d}=\bigcup_{m \in \mathbb{Z}^{d}} \overline{\omega_{m}}$. The vectors $t^{(i)}$ are the basis of the lattice and form the matrix $\mathbf{T}:=\left[t^{(1)}, t^{(2)}, \ldots, t^{(d)}\right]$. We assume

$$
\text { the } d \times d \text { matrix } \mathbf{T} \text { is regular. }
$$

Next we define nodes and edges corresponding to the master cell. Let $\left\{x^{(\kappa)}\right\}_{\kappa=1}^{q}$ $\subset \bar{\omega}$ denote a given set of pairwise different master nodes with periodic copies

$$
x^{(m, \kappa)}:=x^{(\kappa)}+\sum_{i=1}^{d} m_{i} t^{(i)} \quad \forall m \in \mathbb{Z}^{d} .
$$


If there is no ambiguity, we write $(m, \kappa)$, short for $x^{(m, \kappa)}$. As an additional assumption we assume that the reference nodes are chosen such that all points $x^{(m, \kappa)}$, $m \in \mathbb{Z}^{d}, 1 \leq \kappa \leq q$, are pairwise different.

Finally, rods (edges) connecting nodes in the lattice will be defined. Only the rods connecting the nodes in the reference cell with other nodes have to be specified because all other rods are periodic copies of them. A rod connecting a node $x^{(\kappa)}$ with another node $x^{(m, \lambda)}$ is denoted by the triple $(\kappa, m, \lambda) \in \mathcal{G}_{\text {micro }} \times \mathcal{G}_{\text {macro }} \times \mathcal{G}_{\text {micro }}$ with

$$
\mathcal{G}_{\text {micro }}:=\{1,2, \ldots, q\}, \quad \mathcal{G}_{\text {macro }}:=\mathbb{Z}^{d} .
$$

The set of all edges connected to a point $x^{(\kappa)}$ is given by

$$
B_{\kappa}:=\left\{(m, \lambda) \in \mathcal{G}_{\text {macro }} \times \mathcal{G}_{\text {micro }} \text { : There is a rod connecting } x^{(k)} \text { and } x^{(m, \lambda)}\right\}
$$

while the set of all edges connecting a point of type $x^{(\kappa)}$ and points of type $x^{(\cdot, \lambda)}$ is

$$
B_{\kappa, \lambda}:=\left\{m \in \mathcal{G}_{\text {macro }}:(m, \lambda) \in B_{\kappa}\right\} .
$$

Remark 2.1. The periodicity of the lattice implies

$$
n \in B_{\kappa, \lambda} \Longleftrightarrow-n \in B_{\lambda, \kappa} .
$$

In view of Remark 2.1 we introduce (nonuniquely determined) minimal subsets $B_{\mu, \mu}^{-}, B_{\mu, \mu}^{+} \subset B_{\mu, \mu}$ implicitly by the conditions

$$
B_{\mu, \mu}=B_{\mu, \mu}^{-} \cup B_{\mu, \mu}^{+} \quad \text { and } \quad n \in B_{\mu, \mu}^{-} \Leftrightarrow-n \in B_{\mu, \mu}^{+} .
$$

Definition 2.2. The graph of the lattice consists of the nodes $x^{(m, \kappa)},(m, \kappa) \in$ $\mathcal{G}_{\text {macro }} \times \mathcal{G}_{\text {micro }}$ and the edges

$$
((m, \kappa),(n, \lambda)), \quad m-n \in B_{\kappa, \lambda} \cup B_{\lambda, \kappa} .
$$

The following examples are illustrated in Table [1, where $q$ in $\left(x^{(\kappa)}\right)_{\kappa=1}^{q}$ denotes the number of reference nodes and $n e:=\sum_{\kappa=1}^{q} \sum_{\lambda=\kappa}^{q} \sharp B_{\kappa, \lambda}$ the number of reference edges.

Example 2.3. For $1 \leq i \leq d$, let $e_{i}$ denote the canonical unit vector in $\mathbb{R}^{d}$.

(1) The Cartesian lattice with additional diagonal is characterized by the choice

$$
\begin{aligned}
t^{(i)} & :=e_{i}, 1 \leq i \leq d, \\
\omega & =(0,1)^{d}, \quad \mathcal{G}_{\text {micro }}=\{1\}, \quad x^{(1)}=\mathbf{0} \\
B_{1} & =\left\{\left(e_{i}, 1\right): 1 \leq i \leq d\right\} \cup\left\{\left(\sum_{i=1}^{d} e_{i}, 1\right)\right\} .
\end{aligned}
$$

(2) For the definition of the honeycomb lattice in $\mathbb{R}^{2}$ we choose $\omega$ as the convex hull of the points $P_{i}:=\left(\cos \alpha_{i}, \sin \alpha_{i}\right)^{\top}$ with $\alpha_{i}:=(i-1) \pi / 3,1 \leq i \leq 6$. There are two translation (basis) vectors given by

$$
t^{(1)}:=\frac{1}{2}(3, \sqrt{3})^{\top}, \quad t^{(2)}:=(0, \sqrt{3})^{\top} .
$$

The reference nodes are $x_{1}:=(1,0)^{\top}$ and $x_{2}=\left(\cos \frac{\pi}{3}, \sin \frac{\pi}{3}\right)$ with corresponding edges

$$
\begin{aligned}
& \left(1,\left(\begin{array}{c}
1 \\
-1
\end{array}\right), 2\right), \quad\left(1,\left(\begin{array}{l}
0 \\
0
\end{array}\right), 2\right), \quad\left(1,\left(\begin{array}{c}
0 \\
-1
\end{array}\right), 2\right), \\
& \left(2,\left(\begin{array}{l}
0 \\
0
\end{array}\right), 1\right), \quad\left(2,\left(\begin{array}{l}
0 \\
1
\end{array}\right), 1\right), \quad\left(2,\left(\begin{array}{c}
-1 \\
1
\end{array}\right), 1\right) .
\end{aligned}
$$


2.2. Bilinear forms on general lattices. In this subsection, we will describe discrete potentials on general lattices as they arise, e.g., in linear elasticity. We will focus here on general vector potentials while referring for scalar ones to [14].

We start by introducing the space consisting of vector-valued grid functions. With every nodal point $x^{(m, \lambda)}$ we associate a $d$-dimensional vector $u^{(m, \lambda)} \in \mathbb{C}^{d}$. The set of all mappings $u: \mathcal{G}_{\text {micro }} \rightarrow \mathbb{C}^{d}$ forms the set of grid functions corresponding to the master cell, i.e., $\mathcal{S}_{\text {micro }}:=\left(\mathbb{C}^{d}\right)^{\mathcal{G}_{\text {micro }}}$ and the space of grid functions is defined by

$$
\mathcal{S}:=\left(\mathcal{S}_{\text {micro }}\right)^{\mathcal{G}_{\text {macro }}} \cong \mathbb{C}^{\mathbb{Z}^{d} \times\{1,2, \ldots, q\} \times\{1,2, \ldots, d\}} .
$$

If, e.g., the governing physical equations describe linear elasticity, the vector $u_{(m, \kappa)}$ describes the displacements at the node $x^{(m, \kappa)}$. Although the lattice equations presented below might correspond to very different physical applications, we will use the terminology of linear elasticity to denote the arising quantities.

For every $\operatorname{rod}(\lambda, m, \mu) \in B_{\lambda, \mu}$ in the lattice we associate a positive number $E^{(\lambda, m, \mu)}>0$ which is called the modulus of elasticity. For every $E^{(\lambda, m, \mu)}$ we associate a positive semi-definite matrix $\mathbf{E}^{(\lambda, m, \mu)} \in \mathbb{R}^{d \times d}$ being formed by the dyadic products of the rod vector $\left(x^{(\lambda)}, x^{(m, \mu)}\right)$, i.e.,

$$
\mathbf{E}^{(\kappa, m, \lambda)}:=\frac{E^{(\kappa, n, \lambda)}}{\left\|x^{(n, \lambda)}-x^{(\kappa)}\right\|} \frac{x^{(n, \lambda)}-x^{(\kappa)}}{\left\|x^{(n, \lambda)}-x^{(\kappa)}\right\|}\left(\frac{x^{(n, \lambda)}-x^{(\kappa)}}{\left\|x^{(n, \lambda)}-x^{(\kappa)}\right\|}\right)^{\top} .
$$

Here $\langle\cdot, \cdot\rangle$ denotes the Euclidean scalar product in $\mathbb{C}^{d}$ and $\|\cdot\|$ the Euclidean norm. The bilinear form $A: \mathcal{S} \times \mathcal{S} \rightarrow \mathbb{C}$ is defined by

$$
A(u, v):=\sum_{m \in \mathcal{G}_{\text {macro }}} \frac{1}{2} \sum_{\kappa, \lambda \in \mathcal{G}_{\text {micro }}} \sum_{n \in B_{\kappa, \lambda}}\left\langle u_{m+n, \lambda}-u_{m, \kappa}, \mathbf{E}^{(\kappa, n, \lambda)}\left(\bar{v}_{m+n, \lambda}-\bar{v}_{m, \kappa}\right)\right\rangle
$$

for all $u, v \in \mathcal{S}$.

The physical interpretation of this bilinear form in the case of linear elasticity is that the local energy of a rod depends on the modulus of elasticity $E^{(\kappa, n, \lambda)}$ and the component of the displacements $u_{m+n, \lambda}-u_{m, \kappa}$ which is aligned with the rod.

Remark 2.4. We emphasize that the abstract form of the bilinear form $A$ corresponds to physical problems in linear elasticity. In general, we could replace the matrix $\mathbf{E}^{(\kappa, n, \lambda)}$ with a more general positive definite or positive semi-definite matrix. However, our results on the stability of the bilinear form $A$ rely strongly on the definition (2.4) of the matrix $\mathbf{E}^{(\lambda, m, \mu)}$. The assumptions on the geometry of the lattice could possibly be relaxed if we restrict the choice to positive definite matrices instead of positive semi-definite matrices.

We have now all the ingredients for formulating the general lattice equations. In order to obtain solutions with finite energy, we introduce the spaces

$$
\begin{aligned}
\mathcal{S}_{A} & :=\{v \in \mathcal{S}: A(v, v)<\infty\} / Q, \\
\mathcal{S}_{\text {comp }} & :=\left\{v \in \mathcal{S} \mid \exists R>0, \forall(m, \lambda) \in \mathcal{G}_{\text {macro }}^{>R} \times \mathcal{G}_{\text {micro }}: v_{m, \lambda}=0\right\}
\end{aligned}
$$

where $Q$ is the six-dimensional space spanned by the rigid-body motions and

$$
\mathcal{G}_{\text {macro }}^{>R}:=\left\{m \in \mathcal{G}_{\text {macro }}:\|m\|_{\infty}>R\right\} .
$$

The lattice equations are given by seeking for given right-hand side $F \in \mathcal{S}_{\text {comp }}$ a function $u \in \mathcal{S}_{A}$ such that

$$
A(u, v)=F(v), \quad \forall v \in \mathcal{S}_{A} .
$$



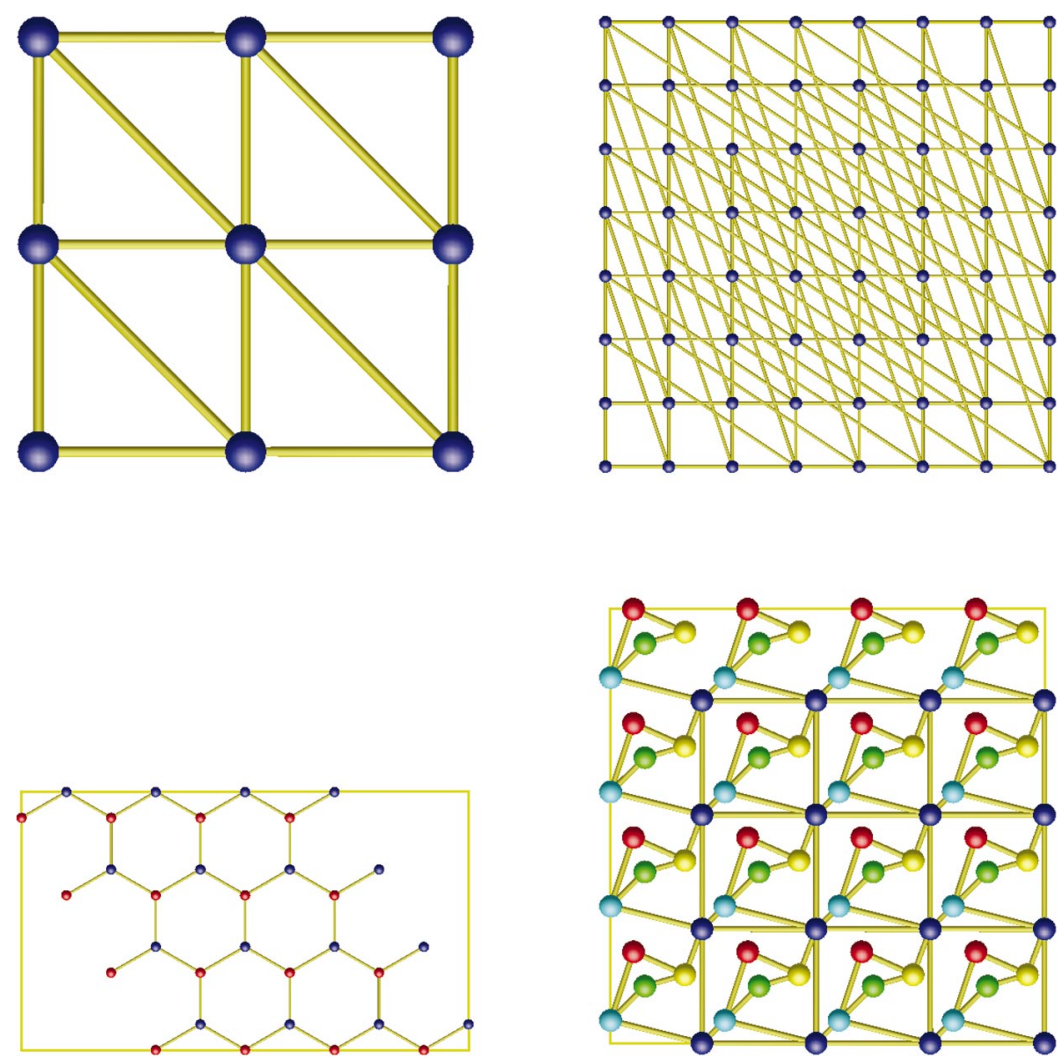

TABLE 1. Two-dimensional lattices. Nodes are marked as balls. All other intersection points stem from edges which lie upon each other but do not intersect in the graph. Nodes of different type are depicted in different colors. The thin yellow frame is drawn for visualisation purpose only. Left top: Cartesian lattice with additional diagonal $q=1$, ne $=3$. Right top: Complicated lattice with $q=1$ and $n e=4$. Left bottom: "Honeycomb lattice" $(q=2, n e=3)$. Right bottom: Lattice with internal microstructure $(q=5, n e=9)$.

The assumption $F \in \mathcal{S}_{\text {comp }}$ allows the application of Fourier techniques for analyzing the solvability of (2.5). We will first motivate and outline the underlying idea of our approach and present the details in the remaining part of this paper.

In the next section, we will compute the matrix-valued symbol $\sigma$ of the bilinear form $A$. Formally, we can write the solution of (2.5) as

$$
u=\mathcal{F}^{-1}\left(\sigma^{-1} \hat{F}\right),
$$

where $\hat{F}$ is the Fourier transform of $F$ and $\mathcal{F}^{-1}$ denotes the inverse Fourier transform.

Standard equilibrium conditions on the function $F$ guarantee that $\hat{F}$ is sufficiently smooth at the origin such that the integral for the inverse Fourier transform 
is well defined in a neighborhood of the origin. For the existence of the global inverse Fourier transform, the inverse symbol $\sigma^{-1}$, in general, may not contain poles outside the origin. This property is equivalent to the condition

$$
\left(\operatorname{det} \sigma(t)=0 \wedge t \in\left[-\pi, \pi\left[^{d}\right) \Leftrightarrow(t=0) .\right.\right.
$$

In [14, it is proved for the case $d=3$ that condition (2.6) implies the existence and uniqueness of (2.5).

In this light, the question of existence and uniqueness of equation (2.5) is reduced to condition (2.6) and we will develop here purely algebraic algorithms for verifying this condition for any given infinite periodic lattice.

2.3. The symbol of discrete vector potentials. The symbol of the bilinearform $A$ in (2.5) is obtained by the Fourier transform which, in the case of vector-valued grid functions $u \in \mathcal{S}$, is applied componentwise, i.e., for all $\kappa \in \mathcal{G}_{\text {micro }}$ and $1 \leq$ $j \leq d$ to the vector $\left(\left(u_{\cdot, \kappa}\right)_{j}\right) \in \mathbb{C}^{\mathcal{G}_{\text {macro }}}$. The symbol is a mapping $\left.\left.\sigma:\right]-\pi, \pi\right]^{d} \rightarrow$

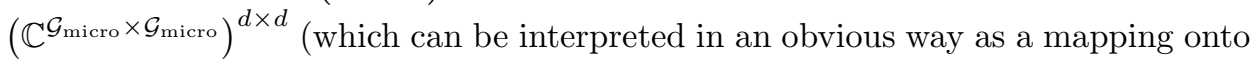
$\left(\mathbb{C}^{d \times d}\right)^{\mathcal{G}_{\text {micro }} \times \mathcal{G}_{\text {micro }}}$ and as a matrix in $\left.\mathbb{C}^{d q \times d q}\right)$.

To compute the symbol of $A$, we will briefly recall some basic facts about the Fourier transform which are well known.

For a grid function $v \in \mathcal{S}$, the Fourier (series) transform is given formally by

$$
\hat{v}_{\kappa, j}(t):=\mathcal{F}\left(\left(v_{\cdot, \kappa}\right)_{j}\right)(t)=\sum_{m \in \mathcal{G}_{\text {macro }}}\left(v_{m, \kappa}\right)_{j} e^{i\langle m, t\rangle}
$$

for all $t \in]-\pi, \pi]^{d}, \kappa \in \mathcal{G}_{\text {micro }}, 1 \leq j \leq d$. The set $\mathcal{S}_{\text {per }}$ consists of all functions $\hat{v}:]-\pi, \pi]^{d} \rightarrow \mathcal{S}_{\text {micro }}$ which have a representation as in (2.7). The inverse Fourier transform is formally defined for functions $\hat{v} \in \mathcal{S}_{\text {per }}$ (again componentwise) by

$$
\left(v_{m, \kappa}\right)_{j}:=\left(\mathcal{F}^{-1} \hat{v}_{\kappa, j}\right)_{m}=(2 \pi)^{-d} \int_{]-\pi, \pi]^{d}} \hat{v}_{\kappa, j}(t) e^{-i\langle m, t\rangle} d t
$$

for all $m \in \mathcal{G}_{\text {macro }}, \kappa \in \mathcal{G}_{\text {micro }}, 1 \leq j \leq d$. Replacing $u$ and $v$ in (2.7) by $\mathcal{F}^{-1} \hat{u}$ and $\mathcal{F}^{-1} \hat{v}$, we get

$$
\begin{aligned}
& \hat{A}(\hat{u}, \hat{v}):=a\left(\mathcal{F}^{-1} \hat{u}, \mathcal{F}^{-1} \hat{v}\right) \\
& =\frac{1}{2} \sum_{\kappa, \lambda \in \mathcal{G}_{\text {micro }}} \sum_{n \in B_{\kappa, \lambda}} \int_{]-\pi, \pi]^{d}}\left\langle\hat{u}_{\lambda, \cdot}(t) e^{-i\langle n, t\rangle}-\hat{u}_{\kappa, \cdot}(t),\right. \\
& \left.\mathbf{E}^{(\kappa, n, \lambda)}\left(e^{i\langle n, t\rangle} \overline{\hat{v}}_{\lambda, \cdot}(t)-\overline{\hat{v}}_{\kappa, \cdot}(t)\right)\right\rangle d t \\
& =\sum_{\kappa, \lambda \in \mathcal{G}_{\text {micro }}} \sum_{j, k=1}^{d} \sum_{n \in B_{\kappa, \lambda}} \int_{-\pi, \pi]^{d}}\left(\hat{u}_{\kappa, j}(t)-\hat{u}_{\lambda, j}(t) e^{-i\langle n, t\rangle}\right) \mathbf{E}_{j, k}^{(\kappa, n, \lambda)} \overline{\hat{v}}_{\kappa, k}(t) d t .
\end{aligned}
$$

Thus, the operator associated with the bilinear form $\hat{A}$ is given by

$$
(\hat{A} \hat{u})_{\kappa, j}:=\sum_{\lambda \in \mathcal{G}_{\text {micro }}} \sum_{k=1}^{d} \sum_{n \in B_{\kappa, \lambda}}\left(\hat{u}_{\kappa, k}(t)-\hat{u}_{\lambda, k}(t) e^{-i\langle n, t\rangle}\right) \mathbf{E}_{j, k}^{(\kappa, n, \lambda)} .
$$

The symbol of this operator is defined by

$$
\sigma_{\mu, \nu}(t):=\delta_{\mu, \nu} \sum_{\lambda \in \mathcal{G}_{\text {micro }}} \sum_{n \in B_{\lambda, \mu}} \mathbf{E}^{(\lambda, n, \mu)}-\sum_{n \in B_{\nu, \mu}} \mathbf{E}^{(\nu, n, \mu)} e^{i\langle n, t\rangle}
$$


and satisfies

$$
a\left(\mathcal{F}^{-1} \hat{u}, \mathcal{F}^{-1} \hat{v}\right)=\sum_{\mu, \nu \in \mathcal{G}_{\text {micro }}}\left\langle\sigma_{\nu, \mu} \hat{u}_{\mu, \cdot}, \hat{v}_{\nu, \cdot}\right\rangle .
$$

For the analysis of the symbol, the representation

$$
\begin{aligned}
\sigma_{\mu, \mu}(t) & =\sum_{\substack{\nu \in \mathcal{G}_{\text {micro }} \\
\nu \neq \mu}} \sum_{n \in B_{\nu, \mu}} \mathbf{E}^{(\nu, n, \mu)}+4 \sum_{n \in B_{\mu, \mu}^{+}} \mathbf{E}^{(\mu, n, \mu)} \sin ^{2} \frac{\langle n, t\rangle}{2} \\
\sigma_{\mu, \nu}(t) & =-\sum_{n \in B_{\nu, \mu}} \mathbf{E}^{(\nu, n, \mu)} e^{i\langle n, t\rangle}
\end{aligned}
$$

will be employed as well.

As already explained at the end of subsection 2.2, the well-posedness of the lattice equations is directly linked to the properties of the symbol and its inverse. The (finite difference) operator associated with the bilinear form $A(\cdot, \cdot)$ is an elliptic operator of second order if the inverse of $\left.\left.\sigma^{-1}:\right]-\pi, \pi\right]^{d} \rightarrow\left(\mathbb{C}^{d \times d}\right)^{\mathcal{G}_{\text {micro }} \times \mathcal{G}_{\text {micro }}}$ has poles, if and only if $t=0$, and the order of this pole is 2 .

In the remaining sections, we will develop algorithms for verifying the connectivity of the lattice and analyzing the poles of the inverse symbol.

\section{Connectivity of LATtices}

In this section, we will develop criteria for the lattice to be connected. Tools from algebra are employed and we recall first the relevant setting. The notation is as introduced in the previous section

Definition 3.1. Two nodes, $(n, \kappa)$ and $(m, \lambda)$, are directly connected iff

$$
m-n \in B_{\kappa, \lambda} \cup B_{\lambda, \kappa} \text {. }
$$

Definition 3.2. Two nodes, $(n, \kappa)$ and $(m, \lambda)$, are connected if there exists a sequence $\left(s^{(i)}\right)_{i=1}^{p}$ of directly connected pairs of nodes satisfying

$$
\begin{aligned}
& \left(s^{(1)}\right)_{1}=(n, \kappa), \quad\left(s^{(p)}\right)_{2}=(m, \lambda), \\
& \left(s^{(i)}\right)_{2}=\left(s^{(i+1)}\right)_{1}, \quad 1 \leq i \leq p .
\end{aligned}
$$

Definition 3.3. A lattice is connected if every node $(n, \kappa)$ is connected to every node $(m, \lambda)$ of the lattice.

In order to check the connectivity of a periodic lattice, we employ tools from ideal theory. First, some notation will be introduced.

Definition 3.4. Let $\mathbf{M} \in \mathbb{Z}^{d \times n}$ denote a matrix and $m^{(i)}$ the $i$ th column vector of $\mathbf{M}$. The span of $\mathbf{M}$ over the $\operatorname{ring} \mathcal{R} \in\{\mathbb{Z}, \mathbb{R}\}$ is given by

$$
\operatorname{span}_{\mathcal{R}} \mathbf{M}=\left\{\sum_{i=1}^{n} \alpha_{i} m^{(i)}: \alpha \in \mathcal{R}^{n}\right\} .
$$

Next, we will give sufficient and necessary conditions for a matrix $\mathbf{M} \in \mathbb{Z}^{d \times n}$ such that, for all $v \in \mathbb{Z}^{d}$, the equation

$$
\mathbf{M} w=v
$$

has a solution $w \in \mathbb{Z}^{n}$. 
Definition 3.5. Let $\mathbf{M} \in \mathbb{Z}^{d \times n}$ and $1 \leq t \leq \min \{d, n\}$. A matrix $\mathbf{S} \in \mathbb{Z}^{t \times t}$ is a $t \times t$ submatrix of $\mathbf{M}$ if there exist indices $1 \leq \ell_{1}<\ell_{2}<\cdots<\ell_{t} \leq d$ and $1 \leq k_{1}<k_{2}<\cdots<k_{t} \leq n$ so that

$$
\mathbf{S}_{i, j}=\mathbf{M}_{\ell_{i}, k_{j}}, \quad 1 \leq i, j \leq t .
$$

The corresponding minor is $\operatorname{det} \mathbf{S}_{i, j}$.

Definition 3.6. Let $\mathbf{M} \in \mathbb{Z}^{d \times n}$. For $1 \leq t \leq \min \{d, n\}$, let $I_{t}(\mathbf{M})$ denote the ideal in $\mathbb{Z}$ generated by all $t \times t$ minors of $\mathbf{M}$.

In other words, the determinants of all submatrices $\mathbf{S} \in \mathbb{Z}^{t \times t}$ form the set $\sigma_{t}=$ $\left\{\Delta_{1}, \Delta_{2}, \ldots, \Delta_{r}\right\}$ of numbers (determinants) $\Delta_{i} \in \mathbb{Z}$. The set

$$
\operatorname{span}_{\mathbb{Z}} \sigma_{t}=\left\{\sum_{i=1}^{r} z_{i} \Delta_{i}: z \in \mathbb{Z}^{r}\right\}
$$

is $I_{t}(\mathbf{M})$. For $t=0$ we put $I_{0}(\mathbf{M})=\mathbb{Z}$.

Theorem 3.7. Let $M \in \mathbb{Z}^{d \times n}$ with $n \geq d$. The following statements (a) and (b) are equivalent.

(a) $I_{d}(\mathbf{M})=\mathbb{Z}$.

(b) For every $v \in \mathbb{Z}^{d}$, the equation

$$
\mathbf{M} w=v
$$

has a solution $w \in \mathbb{Z}^{n}$.

Proof. (a) $\Rightarrow($ b): See [3] Corollary 5.35].

(b) $\Rightarrow(\mathrm{a})$ : If the solution of [3.1) is in $\mathbb{Z}^{d}$, then $I_{t}([\mathbf{M} \mid v])=I_{t}(\mathbf{M})$ for all $0 \leq t \leq d$ and all $v \in \mathbb{Z}^{d}$ (cf. [3, Theorem 5.21]). (The matrix $([\mathbf{M} \mid v]) \in \mathbb{Z}^{d \times(n+1)}$ contains the columns of $\mathbf{M}$ as the first $n$ columns and $v$ as the last column.) Using the elementary properties of determinants, we get the result

$$
I_{t}(\mathbf{M})=\operatorname{span}_{\mathbb{Z}}\left\{I_{t}\left[\mathbf{M} \mid e_{i}\right]: 1 \leq i \leq d\right\},
$$

where $e_{i}$ denotes the $i$ th standard unit vector in $\mathbb{Z}^{d}$. Let $D_{t}(\mathbf{M})$ denote the set of all $t \times t$ minors of a matrix $\mathbf{M}$. Because

$$
D_{t}\left(\left[\mathbf{M} \mid e_{i}\right]\right)=D_{t}(\mathbf{M}) \cup D_{t-1}\left(\mathbf{M}^{(i)}\right),
$$

where $\mathbf{M}^{(i)}$ is the matrix $\mathbf{M}$ after removing the $i$ th row, we obtain

$$
\bigcup_{i=1}^{d} D_{t}\left(\left[\mathbf{M} \mid e_{i}\right]\right)=D_{t}(\mathbf{M}) \cup \bigcup_{i=1}^{d} D_{t-1}\left(\mathbf{M}^{(i)}\right)=D_{t}(\mathbf{M}) \cup D_{t-1}(\mathbf{M}) \text {. }
$$

This leads to

$$
I_{t}(\mathbf{M})=\operatorname{span}_{\mathbb{Z}} D_{t}(\mathbf{M})=\operatorname{span}_{\mathbb{Z}}\left\{D_{t}(\mathbf{M}) \cup D_{t-1}(\mathbf{M})\right\}
$$

and we conclude that

$$
I_{t-1}(\mathbf{M})=\operatorname{span}_{\mathbb{Z}}\left\{D_{t-1}(\mathbf{M})\right\} \subset I_{t}(\mathbf{M}) .
$$

The opposite inclusion $I_{t}(\mathbf{M}) \subset I_{t-1}(\mathbf{M})$ is obvious (cf. [3 p. 28]) and we proved

$$
I_{t-1}(\mathbf{M})=I_{t}(\mathbf{M}) \text {. }
$$


By the induction principle, we obtain

$$
\mathbb{Z}=I_{0}(\mathbf{M})=I_{1}(\mathbf{M})=\cdots=I_{d}(\mathbf{M}) \subseteq \mathbb{Z}
$$

yielding $I_{d}(\mathbf{M})=\mathbb{Z}$.

Remark 3.8. For $n=d$, the condition $I_{d}(\mathbf{M})=\mathbb{Z}$ is equivalent to

$$
|\operatorname{det} \mathbf{M}|=1 \text {. }
$$

Proof. See [3, Corollary 2.21].

For the general case, i.e., $n \geq d$, the verification of the condition $I_{d}(\mathbf{M})=\mathbb{Z}$ is slightly more involved. First, one computes the $d \times d$ minors resulting in the set $\sigma(\mathbf{M})=\left\{\Delta_{1}, \Delta_{2}, \ldots, \Delta_{r}\right\}$. The property $\operatorname{span}_{\mathbb{Z}} \sigma=\mathbb{Z}$ can be evaluated by the algorithm ideal_property(M) (cf. [19, Chapter II.10.2]). For $s \subset \mathbb{Z}$, we put $s^{\bullet}=s \backslash\{0\}$.

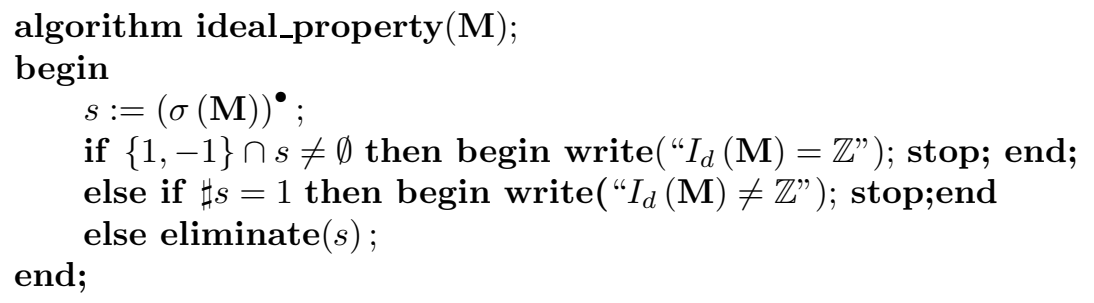

The procedure eliminate is defined below.

procedure eliminate $(s)$;

begin

choose $\Delta_{i} \in s$ with minimal absolute value and $\Delta_{k} \in s \backslash\left\{\Delta_{i}\right\}$;

compute (division with remainder) $p \in \mathbb{Z}$ so that

$$
\left|\Delta_{k}-p \Delta_{i}\right| \leq\left|\Delta_{k}-q \Delta_{i}\right|, \quad \forall q \in \mathbb{Z}
$$

replace $\Delta_{k} \in s$ by $\Delta_{k}-p \Delta_{i}$;

$s:=s^{\bullet}$

if $\{1,-1\} \cap s \neq \emptyset$ then begin write( $I_{d}(\mathbf{M})=\mathbb{Z}$ "); stop; end

else if $\sharp s=1$ then begin write ( $I_{d}(\mathbf{M}) \neq \mathbb{Z}$ "); stop; end else eliminate $(s)$;

end;

The ideal property is strongly related to the connectivity of the lattice as we will see in Theorem 3.9 Recall the definition of the set $B_{\mu, \mu}^{+}$as in (2.3). By introducing a numbering in $B_{\mu, \mu}^{+}=\left\{m^{(1)}, m^{(2)}, \ldots, m^{\left(n_{\mu}\right)}\right\} \subset \mathbb{Z}^{d}$, we may associate the matrix $\mathbf{M}_{\mu, \mu} \in \mathbb{Z}^{d \times n_{\mu}}$ with $B_{\mu, \mu}^{+}$having $m^{(i)}, 1 \leq i \leq n_{\mu}$, as column vectors.

Theorem 3.9. Let $q=1$ (cf. (2.2) ). The lattice is connected if and only if

$$
I_{d}\left(\mathbf{M}_{1,1}\right)=\mathbb{Z} \text {. }
$$

Proof. The lattice is connected if and only if every two nodes $(m, 1)$ and $(n, 1)$ are connected, i.e., if, for all $n \in \mathbb{Z}^{d}$, there exists $w \in \mathbb{Z}^{n_{1}}$ with

$$
m+\sum_{i=1}^{n_{1}} w_{i} m^{(i)}=n
$$


This is equivalent to the statement that, for all $v \in \mathbb{Z}^{d}$, the equation

$$
\mathbf{M} w=v
$$

has a solution in $\mathbb{Z}^{n_{1}}$. Theorem 3.7 implies that this is equivalent to $I_{d}(\mathbf{M})=\mathbb{Z}$.

The case $q>1$ requires some additional notation. For an edge $e=(a, b)$ of the lattice, we denote the edge with opposite orientation by $e^{-}:=(b, a)$.

Definition 3.10. A chain is a sequence $S=\left(s_{i}\right)_{i=1}^{p}$ of edges of the form

$$
s_{i}=\left(\left(m^{(i-1)}, \kappa^{(i-1)}\right),\left(m^{(i)}, \kappa^{(i)}\right)\right) \quad 1 \leq i \leq p
$$

with $\left(m^{(i)}, \kappa^{(i)}\right) \in \mathcal{G}_{\text {macro }} \times \mathcal{G}_{\text {micro }}, 0 \leq i \leq p$. The degree of $S$ is the number of different types of nodes in $S$, i.e.,

$$
\operatorname{deg} S:=\sharp\left\{\kappa^{(i)}: 0 \leq i \leq p\right\} .
$$

A chain is nondegenerate if all nodes $\left(m^{(i)}, \kappa^{(i)}\right), 0 \leq i \leq p$, are of different type, i.e., $\operatorname{deg} S=p+1$.

A chain $S$ is a self-connection of type $\kappa \in \mathcal{G}_{\text {micro }}$ if $\kappa=\kappa^{(0)}=\kappa^{(p)}$. The self-connection is nondegenerate if $\operatorname{deg} S=p$.

For $\ell \in \mathbb{Z}^{d}$, the shift operator $T_{\ell}$ maps an edge $((m, \kappa),(n, \lambda))$ to the shifted edge $((m-\ell, \kappa),(n-\ell, \lambda))$.

Two chains $R=\left(r_{i}\right)_{i=1}^{p}, S=\left(s_{i}\right)_{i=1}^{k}$ can be added if $\left(r_{p}\right)_{2}=(n, \kappa)$ and $\left(s_{1}\right)_{1}=$ $(\tilde{n}, \kappa)$ hold and we write $R+S:=\left(r_{1}, \ldots, r_{p}, T_{\tilde{n}-n}\left(s_{1}\right), \ldots, T_{\tilde{n}-n}\left(s_{k}\right)\right)$. Two sets $\mathfrak{R}, \mathfrak{S}$ of chains are compatible with respect to addition if, for any $r \in \mathfrak{R}, s \in \mathfrak{S}$, the addition $r+s$ is defined and we put

$$
\mathfrak{R}+\mathfrak{S}:=\{R+S: R \in \mathfrak{R}, S \in \mathfrak{S}\} .
$$

The zero-element in the set of self-connections of type $\kappa$ is $((0, \kappa),(0, \kappa))$. by

The multiplication of self-connections $S=\left(s_{i}\right)_{i=1}^{p}$ with integers $\alpha \in \mathbb{Z}$ is defined

$$
\alpha S= \begin{cases}\sum_{i=1}^{\alpha} S & \text { if } \alpha \geq 0 \\ \sum_{i=1}^{-\alpha} S^{-} & \text {if } \alpha<0\end{cases}
$$

where $S^{-}:=\left(s_{p+1-i}^{-}\right)_{i=1}^{p}$. The integer span of a set $\mathfrak{S}=\left\{S_{1}, \ldots, S_{r}\right\}$ of selfconnections is

$$
\operatorname{span}_{\mathbb{Z}} \mathfrak{S}:=\left\{\sum_{i=1}^{r} \alpha_{i} S_{i} \mid \forall 1 \leq i \leq r: \alpha_{i} \in \mathbb{Z}\right\} .
$$


A finite subset $\mathfrak{F} \subset \mathbb{Z}^{d}$ is a frame for a (possibly infinite) set of self-connections $\mathfrak{S}$ if every

$$
S=\left(\left(m^{(i-1)}, \kappa^{(i-1)}\right),\left(m^{(i)}, \kappa^{(i)}\right)\right)_{i=1}^{p} \in \operatorname{span}_{\mathbb{Z}} \mathfrak{S}
$$

satisfies $m^{(p)}-m^{(0)} \in \operatorname{span}_{\mathbb{Z}} \mathfrak{F}$.

Lemma 3.11. Let $q>1$. A lattice is connected if and only if the following conditions are satisfied:

(1) $x^{(1)}$ is connected to any point $x^{(m, 1)}$ for all $m \in \mathbb{Z}^{d}$.

(2) For any $\mu \in \mathcal{G}_{\text {micro }} \backslash\{1\}$, there exists a sequence $\Theta=\left(\kappa_{i}\right)_{i=1}^{p} \subset \mathcal{G}_{\text {micro }}$ such that

$$
\kappa_{1}=1, \quad \kappa_{p}=\mu, \quad B_{\kappa_{i}, \kappa_{i+1}} \neq \emptyset \quad \forall 1 \leq i \leq p-1 .
$$

Proof. The definition of the connectivity of lattices directly implies that both conditions are necessary. To show that these conditions are sufficient, we consider two nodes $x^{(m, \mu)}, x^{(n, \nu)}$, and we construct a connecting chain of the form

$$
x^{(m, \mu)} \rightarrow x^{(\tilde{m}, 1)} \rightarrow x^{(\tilde{n}, 1)} \rightarrow x^{(n, \nu)}
$$

with suitable $\tilde{m}, \tilde{n}$. Let $\Theta=\left(\kappa_{i}\right)_{i=1}^{p}$ denote the sequence as in condition (2) of Lemma 3.11, connecting node 1 with node $\mu$. Choose an associated sequence $m^{(i)} \in$ $\mathcal{G}_{\text {macro }}, 1 \leq i \leq p$, satisfying

$$
m^{(p)}:=m, \quad m^{(i+1)}-m^{(i)} \in B_{\kappa_{i+1}, \kappa_{i}}, \quad 1 \leq i \leq p-1 .
$$

Thus $S_{1}:=\left(\left(m^{(i)}, \kappa_{i}\right),\left(m^{(i-1)}, \kappa_{i-1}\right)\right)_{i=p}^{2}$ is a chain connecting $x^{(m, \mu)}$ and $x^{(\tilde{m}, 1)}$ with $\tilde{m}:=m^{(1)}$. In a similar fashion, a connecting chain $S_{2}$ for the points $x^{(\tilde{n}, 1)}$ and $x^{(n, \nu)}$ is constructed for some $\tilde{n} \in \mathbb{Z}^{d}$. Condition (1) implies that there is a connecting chain $T$ for the nodes $x^{(\tilde{m}, 1)}$ and $x^{(\tilde{n}, 1)}$. Thus, the chain $S_{1}+T+S_{2}$ connects $x^{(m, \mu)}$ and $x^{(n, \nu)}$.

Next, we will derive an algorithm for verifying condition (1) in Lemma 3.11 For $1 \leq i \leq q$, let $\mathfrak{S}_{i}$ denote the set of all nondegenerate self-connections of type 1 and degree $i$ and let $\mathfrak{F}_{i}$ be a frame of $\mathfrak{S}_{i}$. Put $\mathfrak{F}:=\bigcup_{i=1}^{d} \mathfrak{F}_{i}$. Then, condition (1) is satisfied if and only if

$$
\operatorname{span}_{\mathbb{Z}} \mathfrak{F}=\mathbb{Z}^{d}
$$

This property can be verified via algorithm ideal_property. Thus, it remains to derive an algorithm for computing the frame $\mathfrak{F}$. The algorithm is based on a recursion over the degrees of the self-connections.

Obviously, a frame for all self-connections of type $\mu$ and degree 1 is given by $B_{\mu, \mu}^{+}$(cf. [2.3), and we put $\mathfrak{F}_{\mu, \mu}^{(1)}:=B_{\mu, \mu}^{+}, \mu \in \mathcal{G}_{\text {micro. }}$.

By the induction principle we assume that a frame of all self-connections of type $\mu$ and degree $i$ is already computed (and denoted by $\mathfrak{F}_{\mu, \mu}^{(i)}$ ).

For the definition of a frame of all nondegenerate self-connections of degree $i+1$, i.e., of the form

$$
\begin{aligned}
& \mu=: \kappa_{0} \rightarrow \kappa_{1} \rightarrow \kappa_{2} \rightarrow \cdots \kappa_{i} \rightarrow \mu=: \kappa_{i+1} \\
& \text { with mutually different } \kappa_{j} \in \mathcal{G}_{\text {micro }} \backslash\{\mu\}, 1 \leq j \leq i,
\end{aligned}
$$



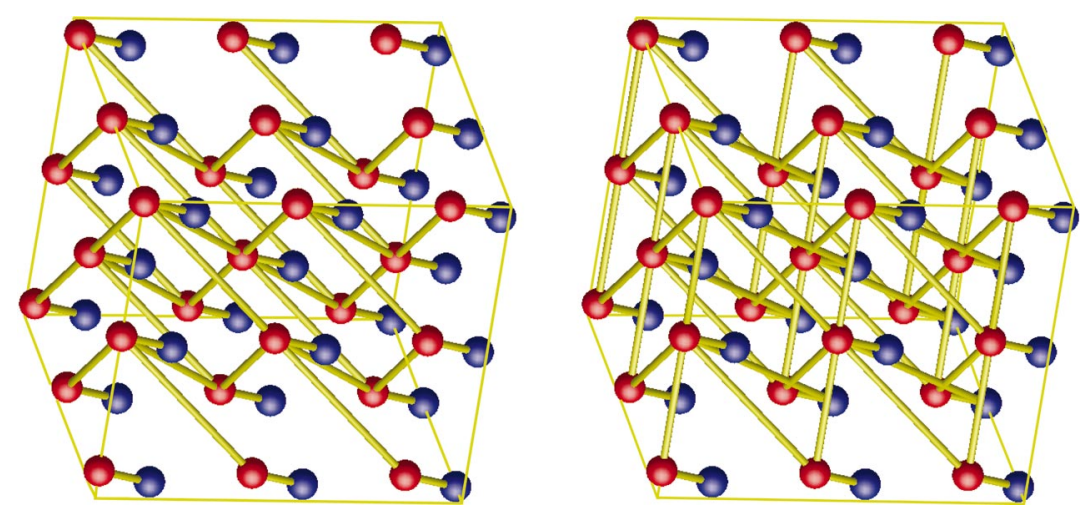

FiguRE 1. Three-dimensional lattices with $q=2$. Left: Lattice with minimal number of reference edges. Right: Minimal lattice which stays connected after removing one arbitrary edge. The thin yellow frame is drawn only for visualization purpose.

we introduce some notation. A connection of the form (3.3) exists if all corresponding sets $B_{\kappa_{j-1}, \kappa_{j}}$ satisfy

$$
B_{\kappa_{j-1}, \kappa_{j}} \neq \emptyset \quad \forall 1 \leq j \leq i+1 .
$$

The set $S_{\mu, \mu}^{(i+1)}$ contains all such connections

$$
S_{\mu, \mu}^{(i+1)}:=\left\{\left(\kappa_{j}\right)_{j=0}^{i+1} \in \mathcal{G}_{\text {micro }}^{i+2} \text { : Conditions (3.3) and (3.4) are satisfied }\right\} .
$$

A frame of all nondegenerate self-connections of degree $i+1$ is defined by

$$
\mathfrak{F}_{\mu, \mu}^{(i+1)}:=\bigcup_{\left(\kappa_{j}\right)_{j=0}^{i+1} \in S_{\mu, \mu}^{(i+1)}}\left(\left\{\sum_{j=1}^{i+1} m^{(j)} \mid m^{(j)} \in B_{\kappa_{j-1}, \kappa_{j}}\right\} \cup \bigcup_{j=0}^{i+1} \mathfrak{F}_{\kappa_{j} \kappa_{j}}^{(i)}\right) .
$$

Obviously, the requested frame in (3.2) can be chosen as $\mathfrak{F}_{1,1}^{(q)}$.

The algorithm check_ideal_property (available via the internet address www.math.unizh.ch/compmath/software.html) is the algorithmic realization of this definition and, in combination with the procedure ideal_property, verifies the connectivity of infinite periodic lattices.

Example 3.12. The algorithm check_ideal_property was employed for solving the following problems:

(a) Find a connected lattice with $q=2$ and $d=3$, where $\sharp\left\{B_{11} \cup B_{12} \cup B_{22}\right\}$ is minimal.

(b) Find a connected lattice with $q=2$ and $d=3$ where $\sharp\left\{B_{11} \cup B_{12} \cup B_{22}\right\}$ is minimal under the condition: After removing an arbitrary edge (and all periodic copies) the lattice stays connected.

The lattices for problems (a) and (b) are depicted in Figure 1 


\section{ANALYSis OF THE SYMBOLS FOR DISCRETE POTENTIALS}

Before we analyze the symbol of discrete vector potentials in subsection 4.2, we will briefly recapitulate the analogue properties of discrete scalar potentials as they arise, e.g., in the physical problem of heat flow through a lattice.

4.1. Analysis of the symbols for discrete scalar potentials. In the case of heat flow through a lattice, the value of the grid function at a nodal point represents the temperature at that point and is a scalar quantity. The space of grid functions is given by

$$
\mathcal{S}_{1}:=\left\{u: \mathcal{G}_{\text {macro }} \times \mathcal{G}_{\text {micro }} \rightarrow \mathbb{C}\right\} .
$$

The conductivity of a rod $(\kappa, m, \lambda)$ is characterized by a positive number $\hat{a}^{(\kappa, m, \lambda)}>$ 0 and, for scaling reasons, we set $a^{(\kappa, m, \lambda)}:=\hat{a}^{(\kappa, m, \lambda)} /\left\|x^{(\kappa)}-x^{(m, \lambda)}\right\|$. The bilinear form in this case has the general form

$$
A_{1}(u, v):=\sum_{m \in \mathcal{G}_{\text {macro }}} \frac{1}{2} \sum_{\kappa, \lambda \in \mathcal{G}_{\text {micro }}} \sum_{n \in B_{\kappa, \lambda}}\left(u_{m+n, \lambda}-u_{m, \kappa}\right) a^{(\kappa, n, \lambda)}\left(\bar{v}_{m+n, \lambda}-\bar{v}_{m, \kappa}\right),
$$

while the symbol $\left.\left.\sigma_{1}:\right]-\pi, \pi\right]^{d} \rightarrow \mathbb{C}^{\mathcal{G}_{\text {micro }} \times \mathcal{G}_{\text {micro }}}$ is given by

$$
\sigma_{1}(t)_{\mu, \nu}:= \begin{cases}\sum_{\lambda \in \mathcal{G}_{\text {micro }} \backslash\{\mu\}} \sum_{n \in B_{\lambda, \mu}} a^{(\lambda, n, \mu)}+4 \sum_{n \in B_{\mu, \mu}^{+}} a^{(\mu, n, \mu)} \sin ^{2} \frac{\langle n, t\rangle}{2} & \mu=\nu, \\ -\sum_{n \in B_{\nu, \mu}} a^{(\nu, n, \mu)} e^{-i\langle n, t\rangle} & \mu \neq \nu,\end{cases}
$$

for all $\mu, \nu \in \mathcal{G}_{\text {micro }}$ and $B_{\mu, \mu}^{+}$as in $(2.3)$.

In [14, Lemma 3.2], the stability of the symbol was proved under the only assumption that the lattice is connected.

Theorem 4.1. Suppose the lattice is connected.

(1) There exist constants $c_{1}$ and $C_{1}>0$ which depend only on $q$ and the coefficients $a^{(\mu, n, \nu)}$ such that

$$
\left.\left.c_{1}\|t\|^{2} \leq \operatorname{det} \sigma_{1}(t) \leq C_{1}\|t\|^{2} \quad \forall t \in\right]-\pi, \pi\right]^{d} .
$$

(2) There exist constants $c_{2}$ and $C_{2}>0$ which depend only on $q$ and the coefficients $a^{(\mu, n, \nu)}$ such that the inverse symbol satisfies, for all $\left.\left.t \in\right]-\pi, \pi\right]^{d} \backslash\{0\}$ and $i, j \in \mathcal{G}_{\text {micro }}$, the estimate

$$
c_{2}\|t\|^{-2} \leq\left(\sigma_{1}^{-1}\right)_{i, j}(t) \leq C\|t\|^{-2} .
$$

The following examples show that an analogous theorem cannot hold in the case of vector potentials which are of the form (2.5).

Example 4.2. Let $d=2, q=1$, and $B_{1,1}^{+}=\left\{(1,0)^{\top},(0,1)^{\top}\right\}$. Explicit calculations yield

$$
\operatorname{det} \sigma(t)=16 E^{(1,(1,0), 1)} E^{(1,(0,1), 1)} \sin ^{2} \frac{t_{1}}{2} \sin ^{2} \frac{t_{2}}{2}
$$

and the zeroes are lying on the lines $(\{0\} \times \mathbb{R}) \cup(\mathbb{R} \times\{0\}) \cap]-\pi, \pi]^{2}$. 


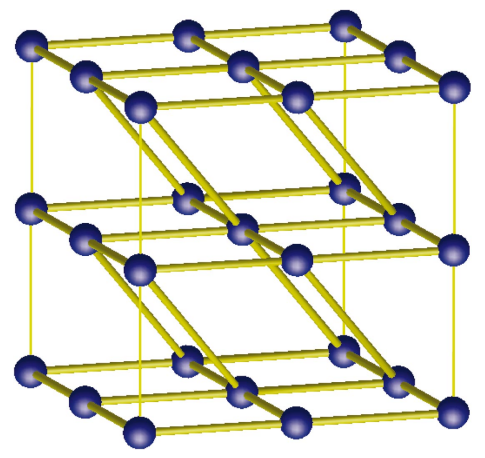

FIgURE 2. Connected but nonrigid, three-dimensional lattice with $q=1$.

Example 4.3. Let $d=2, q=1$, and $B_{1,1}^{+}=\left\{(1,0)^{\top},(0,1)^{\top},(2,0)^{\top}\right\}$. Explicit calculations yield

$$
\operatorname{det} \sigma(t)=16 E^{(1,(0,1), 1)}\left(E^{(1,(1,0), 1)}+4 E^{(1,(2,0), 1)} \sin ^{2} \frac{t_{1}}{2}\right) \sin ^{2} \frac{t_{1}}{2} \sin ^{2} \frac{t_{2}}{2}
$$

and the zeroes of $\operatorname{det} \sigma(t)$ are as in Example 4.2

In both examples, the zeroes of the determinant of the symbol are lying on the union of linear manifolds through the origin. The next example shows that the set of zeroes of the determinant can be a discrete set.

Example 4.4. Let $d=3$ and $q=1$. Let

$$
B_{1,1}^{+}:=\left\{m^{(i)}: 1 \leq i \leq 5\right\}:=\left\{\left(\begin{array}{l}
4 \\
0 \\
0
\end{array}\right),\left(\begin{array}{l}
0 \\
1 \\
0
\end{array}\right),\left(\begin{array}{l}
0 \\
0 \\
1
\end{array}\right),\left(\begin{array}{l}
1 \\
1 \\
1
\end{array}\right),\left(\begin{array}{l}
2 \\
1 \\
3
\end{array}\right)\right\} .
$$

From the procedure check_ideal_property we conclude that this mesh is connected. Lemma 4.8 below implies

$$
\operatorname{det} \sigma(t)=\sum_{1 \leq i<j<k \leq 5} \gamma_{i, j, k} \sin ^{2} \frac{\left\langle m^{(i)}, t\right\rangle}{2} \sin ^{2} \frac{\left\langle m^{(j)}, t\right\rangle}{2} \sin ^{2} \frac{\left\langle m^{(k)}, t\right\rangle}{2}
$$

with some positive numbers $\gamma_{i, j, k}$. Some combinatorial manipulations result in

$$
\left.\{t \in]-\pi, \pi]^{3}: \operatorname{det} \sigma(t)=0\right\}=\left\{(0,0,0)^{\top},(\pi / 2,0,0)^{\top}\right\} .
$$

The physical interpretation of a discrete nonzero root $t_{s}$ of the symbol is as follows. There exists a nonzero vector $\varphi \in \mathcal{S}_{\text {micro }}$ such that $\left\langle\sigma\left(t_{s}\right) \varphi, \varphi\right\rangle=0$. Hence, the corresponding displacement field $u_{m}:=e^{\left\langle m, t_{s}\right\rangle} \varphi$, induces no strain energy, i.e., preserves the lengths of connecting rods. The mesh is depicted in Figure 2

4.2. Analysis of the symbol of discrete vector potentials. It turns out that the analysis of the symbol for vector potentials is much more involved than for scalar potentials. In [14], it is shown that Theorem 4.1] also holds for the discrete vector potentials under consideration, provided (a) the lattice is connected and (b) the lattice is locally rigid (cf. [14]). Condition (b), however, is only sufficient and 
the algorithm presented in [14, in general, does not answer the question of local rigidity in finite time.

We will present here an algorithm which, for the case $q=1$, serves as a sufficient and necessary condition such that Theorem 4.1 holds for the vector potentials under consideration as well. The restriction to the case $q=1$ means that the master cell contains exactly one node.

For $q=1$, the symbol has the form

$$
\sigma(t)=4 \sum_{n \in B_{1,1}^{+}} \mathbf{E}^{(1, n, 1)} \sin ^{2} \frac{\langle n, t\rangle}{2} .
$$

Definition 4.5. Let $q=1$, and let the lattice be characterized by the set $B_{1,1}^{+}$. The lattice is rigid iff the condition

$$
\left.(\exists t \in]-\pi, \pi]^{d}: \quad \operatorname{det} \sigma(t)=0\right) \Leftrightarrow(t=0)
$$

holds.

In this section, we develop an algorithm for verifying the rigidity of a lattice with $q=1$.

Some necessary conditions for (4.2) are described below in Corollaries 4.10, 4.11 and Proposition 4.12.

In order to derive a representation of the determinant of the symbol, we will introduce some additional notation.

Notation 4.6. For a matrix $\mathbf{B}$, the $i$ th column vector is denoted by $b^{(i)}$.

Let $\mathbf{T} \in \mathbb{R}^{d \times d}$ denote the matrix formed by the basis of the lattice $t^{(i)}, 1 \leq i \leq d$, (cf. (2.1)), i.e., $\mathbf{T}=\left[t^{(1)}, t^{(2)}, \ldots, t^{(d)}\right]$.

Notation 4.7. The space $\mathbb{Z}^{d \times d} / \pi$ is the quotient space of $\mathbb{Z}^{d \times d}$, where all matrices which have the same set of column vectors (only the ordering of the column vectors may differ) form the equivalence classes. For $k \geq d$ and a subset $S=\left\{m^{(1)}, m^{(2)}, \ldots, m^{(k)}\right\} \subset \mathbb{Z}^{d}$, we introduce the set of matrices

$$
\mathcal{M}(S):=\left\{\mathbf{M} \in \mathbb{Z}^{d \times d} / \pi: \mathbf{M} \text { is regular and, for all } 1 \leq i \leq d, m^{(i)} \in S\right\} .
$$

For $m \in \mathbb{Z}^{d}$, we write $\hat{m}$, short for $\mathbf{T} m$, and, for a matrix $\mathbf{M}$ with column vectors $m^{(i)}$, we define $\hat{\mathbf{M}}$ as the matrix with column vectors $\widehat{m^{(i)}}$.

Lemma 4.8. The determinant of the symbol has the representation

$$
\operatorname{det} \sigma(t)=4^{d} \sum_{\mathbf{M} \in \mathcal{M}\left(B_{1,1}^{+}\right)} \gamma_{\mathbf{M}}(\operatorname{det} \hat{\mathbf{M}})^{2}
$$

where the nonnegative numbers $\gamma_{\mathbf{M}}$ are given by

$$
\gamma_{\mathbf{M}}=\prod_{i=1}^{d} \frac{E^{\left(1, m^{(i)}, 1\right)}}{\left\|\widehat{m^{(i)}}\right\|^{3}} \sin ^{2} \frac{\left\langle m^{(i)}, t\right\rangle}{2} .
$$


Proof. From $\lambda=\kappa=1$ we conclude that $x^{(n, \lambda)}-x^{(\kappa)}=\hat{n}$. Hence, $\mathbf{E}^{(1, n, 1)}=$ $\frac{E^{(1, n, 1)}}{\|\hat{n}\|^{3}} \hat{n} \hat{n}^{\top}$. We introduce the abbreviation $e_{n}:=e_{n}(t):=4 E^{(1, n, 1)} /\|\hat{n}\|^{3} \sin ^{2} \frac{\langle n, t\rangle}{2}$ to obtain

$$
\sigma(t):=\sum_{n \in B_{1,1}^{+}} e_{n} \hat{n} \hat{n}^{\top}
$$

Expanding the determinant of the symbol with respect to the first row yields

$$
\operatorname{det} \sigma(t)=\sum_{i=1}^{d}(-1)^{i+1} \sum_{n \in B_{1,1}^{+}} e_{n} \hat{n}_{1} \hat{n}_{i} \operatorname{det}\left(\sum_{m \in B_{1,1}^{+}} e_{m} \hat{m}^{(1)}\left(\hat{m}^{(i)}\right)^{\top}\right) \text {, }
$$

where, for a vector $w \in \mathbb{R}^{k}$ and given set of integers $\iota \subset \mathbb{N}$, we set $w^{\iota}:=\left(w_{i}\right)_{\substack{i=1 \\ i \notin \iota}}^{k}$. Lemma 4.9 implies

$$
\begin{aligned}
\operatorname{det} \sigma(t)= & \sum_{i=1}^{d}(-1)^{i+1} \sum_{\ell_{1} \in B_{1,1}^{+}} e_{\ell_{1}}\left(\hat{\ell}_{1}\right)_{1}\left(\hat{\ell}_{1}\right)_{i} \\
& \times \sum_{\ell_{2}, \ldots, \ell_{d} \in B_{1,1}^{+}}\left(\prod_{j=2}^{d}\left(\hat{\ell}_{j}\right)_{j} e_{\ell_{j}}\right) \operatorname{det}\left[\hat{\ell}_{2}^{(i)}, \ldots, \hat{\ell}_{d}^{(i)}\right] \\
= & \sum_{\ell_{1} \in B_{1,1}^{+}} e_{\ell_{1}}\left(\hat{\ell}_{1}\right)_{1} \sum_{\ell_{2}, \ldots, \ell_{d} \in B_{1,1}^{+}}\left(\prod_{j=2}^{d}\left(\hat{\ell}_{j}\right)_{j} e_{\ell_{j}}\right) \\
& \times \sum_{i=1}^{d}(-1)^{i+1}\left(\hat{\ell}_{1}\right)_{i} \operatorname{det}\left[\hat{\ell}_{2}^{(i)}, \ldots, \hat{\ell}_{d}^{(i)}\right] \\
= & \sum_{\ell_{1}, \ldots, \ell_{d} \in B_{1,1}^{+}}\left(\prod_{j=1}^{d}\left(\hat{\ell}_{j}\right)_{j} e_{\ell_{j}}\right) \operatorname{det}\left[\hat{\ell}_{1}, \ldots, \hat{\ell}_{d}\right] .
\end{aligned}
$$

Because determinants are alternating multilinear forms, we obtain

$$
\operatorname{det} \sigma(t)=\sum_{\left[\ell_{1}, \ell_{2}, \ldots, \ell_{d}\right] \in \mathcal{M}\left(B_{1,1}^{+}\right)}\left(\prod_{j=1}^{d} e_{\ell_{j}}\right) \operatorname{det}^{2}\left[\hat{\ell}_{1}, \hat{\ell}_{2}, \ldots, \hat{\ell}_{d}\right] .
$$

The proof of the auxiliary Lemma 4.9 is elementary linear algebra and we include the proof for completeness.

Lemma 4.9. For $k \in\{1,2, \ldots, d\}$, let $M_{k}:=\{1,2, \ldots, k\}$ and $N_{k} \subset M_{d}$ with $\sharp N_{k}=k$. Then, for $k \leq d-1$,

$$
\begin{aligned}
\operatorname{det} & \sum_{\ell \in B_{1,1}^{+}} e_{\ell} \hat{\ell}^{M_{k}}\left(\hat{\ell}^{N_{k}}\right)^{\top} \\
& =\sum_{\ell_{k+1}, \ell_{k+2}, \ldots, \ell_{d} \in B_{1,1}^{+}}\left(\prod_{j=k+1}^{d}\left(\hat{\ell}_{j}\right)_{j} e_{\ell_{j}}\right) \operatorname{det}\left[\hat{\ell}_{k+1}^{N_{k}}, \hat{\ell}_{k+2}^{N_{k}}, \ldots, \hat{\ell}_{d}^{N_{k}}\right] .
\end{aligned}
$$


Proof. The proof is by induction from $k=d-1$ down to 1 . For $k=d-1$, we obtain that the determinant (of the $1 \times 1$-matrix $\sum_{\ell \in B_{1,1}^{+}} e_{\ell} \hat{\ell}^{M_{d-1}}\left(\hat{\ell}^{N_{d-1}}\right)^{\top}$ ) has the representation as in (4.5)

$$
\operatorname{det} \sum_{\ell \in B_{1,1}^{+}} e_{\ell} \hat{\ell}^{M_{d-1}}\left(\hat{\ell}^{N_{d-1}}\right)^{\top}=\sum_{\ell \in B_{1,1}^{+}} e_{\ell} \hat{\ell}_{d} \hat{\ell}_{j}
$$

with $\{j\}=M_{d} \backslash N_{d-1}$.

Assume the assertion is proved for $k+1$. Expanding the determinant with respect to the first row yields

$$
\begin{aligned}
\operatorname{det} & \sum_{\ell \in B_{1,1}^{+}} e_{\ell} \hat{\ell}^{M_{k}}\left(\hat{\ell}^{N_{k}}\right)^{\top} \\
& =\sum_{\substack{i=1 \\
i \notin N_{k}}}^{d}(-1)^{i} \sum_{\ell \in B_{1,1}^{+}} e_{\ell} \hat{\ell}_{k+1} \hat{\ell}_{i} \operatorname{det}\left(\sum_{m \in B_{1,1}^{+}} e_{m} \hat{m}^{M_{k+1}}\left(\hat{m}^{N_{k+1}^{(i)}}\right)\right)^{\top}
\end{aligned}
$$

with $N_{k+1}^{(i)}:=N_{k} \cup\{i\}$. We employ the result for $k+1$ to obtain

$$
\begin{aligned}
\operatorname{det} \sum_{\ell \in B_{1,1}^{+}} e_{\ell} \hat{\ell}^{M_{k}}\left(\hat{\ell}^{N_{k}}\right)^{\top} & \\
= & \sum_{\substack{\ell_{k+1}, \ell_{k+2}, \ldots, \ell_{d} \in B_{1,1}^{+} \\
d}}\left(\prod_{j=k+1}^{d}\left(\hat{\ell}_{j}\right)_{j} e_{\ell_{j}}\right) \\
& \quad \times \sum_{\substack{i=1 \\
i \notin N_{k}}}^{d}(-1)^{i}\left(\hat{\ell}_{k+1}\right)_{i} \operatorname{det}\left[\hat{\ell}_{k+2}^{N_{k+1}^{(i)}}, \hat{\ell}_{k+3}^{N_{k+1}^{(i)}}, \ldots, \hat{\ell}_{d}^{N_{k+1}^{(i)}}\right] \\
= & \sum_{\ell_{k+1}, \ell_{k+2}, \ldots, \ell_{d} \in B_{1,1}^{+}}\left(\prod_{j=k+1}^{d}\left(\hat{\ell}_{j}\right)_{j} e_{\ell_{j}}\right) \operatorname{det}\left[\hat{\ell}_{k+1}^{N_{k}}, \hat{\ell}_{k+2}^{N_{k}}, \ldots, \hat{\ell}_{d}^{N_{k}}\right]
\end{aligned}
$$

and this is the assertion for $k$.

The determinant of the symbol is zero if all summation terms in (4.3) vanish or $\mathcal{M}\left(B_{1,1}^{+}\right)$is the empty set. $\mathcal{M}\left(B_{1,1}^{+}\right)$is the empty set, if all subsets $\left(B_{1,1}^{+}\right)^{\prime} \subset B_{1,1}^{+}$ with $\sharp\left(B_{1,1}^{+}\right)^{\prime}=d$ are linearly dependent.

Corollary 4.10. The connectivity of the lattice implies $\mathcal{M}\left(B_{1,1}^{+}\right) \neq \emptyset$.

For a given set $B_{1,1}^{+}$, the recursive procedure check_det verifies the condition (4.6)

$$
\left.\left.\left(\exists s^{\star} \in\right]-1,1\right]^{d} \quad \forall \mathbf{M} \in \mathcal{M}\left(B_{1,1}^{+}\right) \quad \exists m \in \operatorname{Col}(\mathbf{M}): \frac{\left\langle m, s^{\star}\right\rangle}{2} \in \mathbb{Z}\right) \Leftrightarrow\left(s^{\star}=0\right),
$$

where $\mathrm{Col}(\cdot)$ denotes the set of column vectors of a matrix. Clearly, condition (4.6) is equivalent to condition (4.2). 
The procedure is called by check_det $\left(B_{1,1}^{+}, 1\right)$ and is defined by

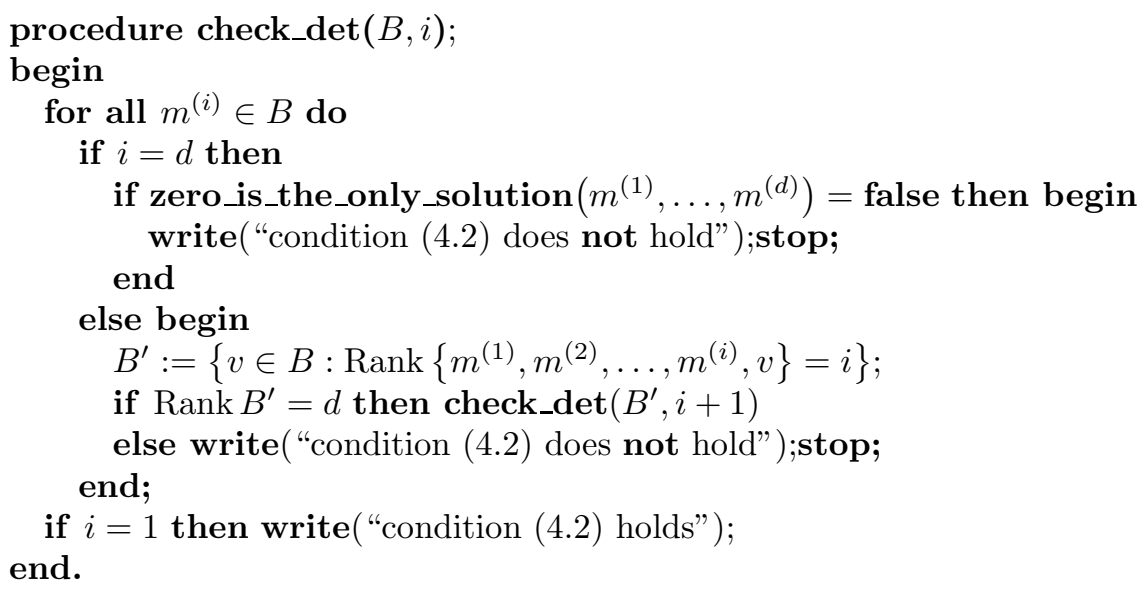

The function zero_is_the_only_solution is defined by

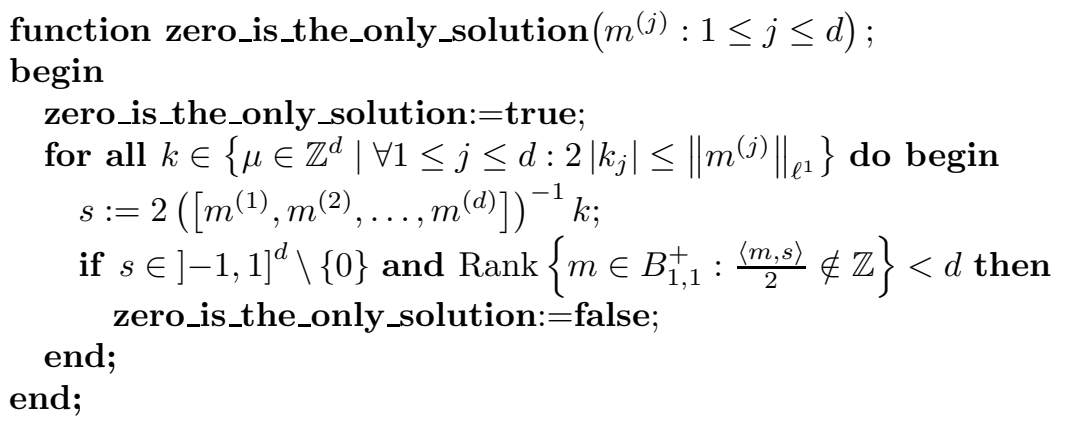

A PASCAL implementation of procedure check_det is available in electronic form from the internet address www.math.unizh.ch/compmath/software.html.

Corollary 4.11. Condition (4.2) implies $\sharp B_{1,1}^{+} \geq 2 d-1$.

Proof. Indirect: Let $\sharp B_{1,1}^{+} \leq 2 d-2$. Choose any subset $\left(B_{1,1}^{+}\right)^{\prime} \subset B_{1,1}^{+}$with $\sharp B_{1,1}^{+} \leq$ $d-1$. Hence, there exists $t \in]-\pi, \pi]^{d} \backslash\{0\}$ with $\langle m, t\rangle=0$ for all $m \in\left(B_{1,1}^{+}\right)^{\prime}$. Any matrix $\mathbf{M} \in \mathcal{M}\left(B_{1,1}^{+}\right)$contains at least one row $m \in\left(B_{1,1}^{+}\right)^{\prime}$ and, thus, condition (4.2) is violated.

The following proposition provides a criterion to determine whether the case $k \neq 0$ is irrelevant in the function zero_is_the_only_solution.

Proposition 4.12. Let $\mathbf{M} \in \mathbb{Z}^{d \times d}$ satisfy $|\operatorname{det} \mathbf{M}|=1$. Then,

$$
\left.\{t \in]-1,1]^{d} \mid \forall m \in \operatorname{Col}(\mathbf{M}): \frac{\langle m, t\rangle}{2} \in \mathbb{Z}\right\}=\{0\} .
$$

Proof. Condition $\operatorname{det} \mathbf{M}= \pm 1$ implies that $\left(\mathbf{M}^{\top}\right)^{-1} \in \mathbb{Z}^{d \times d}$. For any $k \in \mathbb{Z}^{d}$, the solution of $\mathbf{M}^{\top} t=2 k$ has only even components and the condition $\left.\left.t \in\right]-1,1\right]^{d}$ yields $t=0$. 


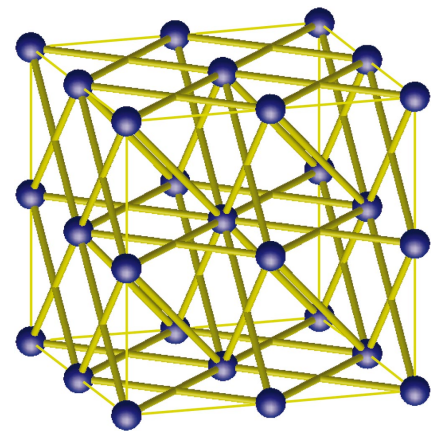

Figure 3. Minimal three-dimensional lattice which is connected and rigid.

Example 4.13. Let $d=2, q=1$, and $B_{1,1}^{+}=\left\{(1,0)^{\top},(0,1)^{\top},(1,1)^{\top}\right\}$. Procedure check_det implies that $\operatorname{det} \sigma(t)=0$ if and only if $t=0$.

Example 4.14. Consider the Cartesian lattice as in Example 2.3

(1) For $d=2$, the determinant of the symbol is zero if and only if $t=0$.

(2) For $d \geq 3$, we have $\sharp B_{1,1}^{+}=d+1<2 d-1$ and the determinant of the symbol has zeroes for some $t \in]-\pi, \pi]^{d} \backslash\{0\}$.

In three dimensions, at least five rods are necessary so that the determinant of the symbol is zero if and only if $t=0$. Let us consider the Cartesian lattice in three dimensions and define the set of all possible connections which stay within one cell

$$
\mathcal{C}:=\left\{\left(\begin{array}{l}
1 \\
0 \\
0
\end{array}\right),\left(\begin{array}{l}
0 \\
1 \\
0
\end{array}\right),\left(\begin{array}{l}
0 \\
0 \\
1
\end{array}\right),\left(\begin{array}{l}
1 \\
1 \\
0
\end{array}\right),\left(\begin{array}{l}
1 \\
0 \\
1
\end{array}\right),\left(\begin{array}{l}
0 \\
1 \\
1
\end{array}\right),\left(\begin{array}{l}
1 \\
1 \\
1
\end{array}\right)\right\} .
$$

The assertions of the following example are derived by applying procedure check det.

Example 4.15. (a) For $B_{1,1}^{+}=\mathcal{C}$, condition (4.2) is satisfied.

(b) For any $B_{1,1}^{+} \subset \mathcal{C}$ with $\sharp B_{1,1}^{+}=5$, condition (4.2) is violated.

(c) For

$$
B_{1,1}^{+}=\left\{\left(\begin{array}{l}
1 \\
0 \\
1
\end{array}\right),\left(\begin{array}{c}
1 \\
0 \\
-1
\end{array}\right),\left(\begin{array}{l}
0 \\
1 \\
1
\end{array}\right),\left(\begin{array}{c}
0 \\
1 \\
-1
\end{array}\right),\left(\begin{array}{l}
1 \\
1 \\
1
\end{array}\right)\right\}
$$

condition (4.2) is satisfied. This lattice is depicted in Figure 3.

Example 4.16 (Exotic lattice). Choose $d=2$ and $q=1$. Put $B_{1,1}^{+}=\left\{(8,63)^{\top}\right.$, $\left.(1,8)^{\top},(a, b)^{\top}\right\}$. The lattice is connected since det $\left[\begin{array}{cc}8 & 1 \\ 63 & 8\end{array}\right]=1$. Choose $a$ and $b$ so that

$$
\operatorname{det}\left[\begin{array}{cc}
8 & a \\
63 & b
\end{array}\right]=1 \text { and } \operatorname{det}\left[\begin{array}{cc}
1 & a \\
8 & b
\end{array}\right]=1
$$

(The unique solution is $a=-7$ and $b=-55$.) Then, the determinant of the symbol is zero if and only if $t=0$. 

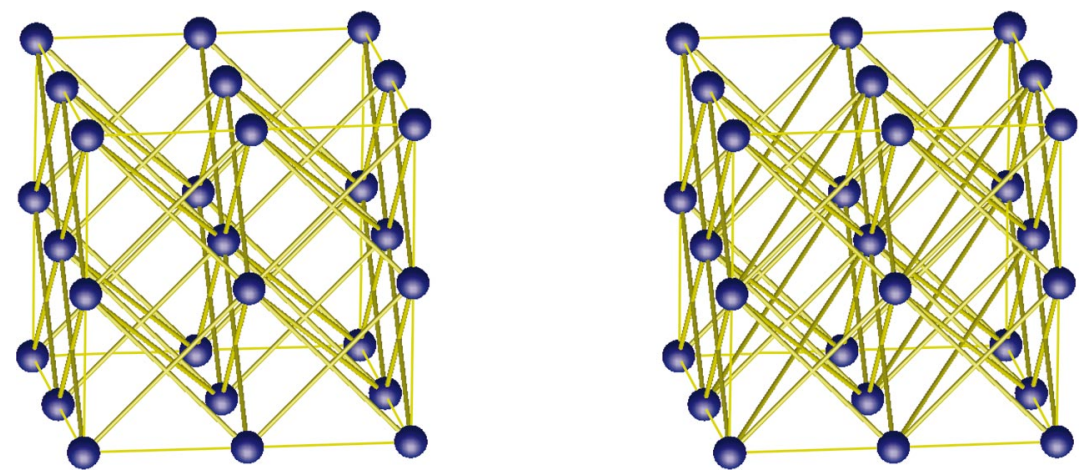

Figure 4. Three-dimensional lattices with $q=1$. Left: Minimal lattice which is connected and rigid. Right: Minimal lattice which stays rigid after removing one arbitrary edge.

Example 4.17. The algorithm check_det was employed for solving the following problems.

(a) Find a connected lattice with $q=1, d=3$ satisfying [4.2), where $n e:=\sharp B_{11}^{+}$ is minimal.

(b) Find a lattice with $q=1, d=3$ satisfying (4.2) where $n e:=\sharp B_{11}^{+}$is minimal under the condition: After removing an arbitrary edge (and all periodic copies), the lattice stays connected.

The lattices for problems (a) and (b) are depicted in Figure 4

Lemma 4.18. Let condition (4.2) be satisfied. Then, $\operatorname{det} \sigma(t)$ has a zero of order $2 d$ at $t=0$.

Proof. Let $S_{1}$ denote the unit sphere in $\mathbb{R}^{d}$. We define $\gamma=\gamma\left(B_{1,1}^{+}\right)$by

$$
\gamma:=\inf _{\xi \in S_{1}} \sup _{\mathbf{M} \in \mathcal{M}\left(B_{1,1}^{+}\right)} \inf _{1 \leq i \leq d}\left|\left\langle m^{(i)}, \xi\right\rangle\right| .
$$

First, we will prove $\gamma>0$.

Clearly $\gamma \geq 0$. Because $S_{1}$ is compact, it is sufficient to show that, for all $\xi \in S_{1}$, there is $\mathbf{M} \in \mathcal{M}\left(B_{1,1}^{+}\right)$such that, for all $1 \leq j \leq d$,

$$
\left\langle m^{(j)}, \xi\right\rangle \neq 0 \text {. }
$$

Because $\xi \in S_{1} \subset[-\pi, \pi]^{d} \backslash\{0\}$, we know that the determinant of the symbol is positive. Formula (4.3) along with condition (4.2) implies that there exists $\mathbf{M} \in$ $\mathcal{M}\left(B_{1,1}^{+}\right)$such that

$$
\frac{\left\langle m^{(j)}, \xi\right\rangle}{2} \neq 0, \quad \forall 1 \leq j \leq d .
$$

Thus, the auxiliary statement $\gamma>0$ is proven.

Let $t \in[-\pi, \pi]^{d} \backslash\{0\}$. Choose $\xi \in S_{1}$ so that $t=\|t\| \xi$ and $\mathbf{M}=\mathbf{M}(\xi)$ as before. Hence,

with

$$
C\|t\| \geq\left|\left\langle m^{(i)}, t\right\rangle\right| \geq \gamma\|t\|, \quad \forall 1 \leq i \leq d
$$

$$
C:=\max _{m \in B_{1,1}^{+}}\|m\| .
$$


For sufficiently small $\|t\|$, we can estimate

$$
\begin{aligned}
\gamma_{\mathbf{M}} & \geq c \prod_{i=1}^{d} \frac{\mathbf{E}^{\left(1, m^{(i)}, 1\right)}}{\left\|\widehat{m^{(i)}}\right\|}\left(\left\|\widehat{m^{(i)}}\right\|^{-1} c \gamma\|t\|\right)^{2} \\
& \geq c(c \gamma)^{2 d} \min _{1 \leq i \leq d}\left(\mathbf{E}^{\left(1, m^{(i)}, 1\right)}\left\|\widehat{m^{(i)}}\right\|^{-3}\right)^{d}\|t\|^{2 d},
\end{aligned}
$$

resulting in

$$
\operatorname{det} \sigma(t) \geq\left\{c(\operatorname{det} \hat{\mathbf{M}})^{2}\left((2 \gamma c)^{2} \min _{1 \leq i \leq d} \frac{\mathbf{E}^{\left(1, m^{(i)}, 1\right)}}{\left\|\widehat{m^{(i)}}\right\|^{3}}\right)^{d}\right\}\|t\|^{2 d} .
$$

The matrix $\hat{\mathbf{M}}$ depends on $\xi$ and therefore on $t$. To obtain an estimate of the expression in brackets $\{\ldots\}$ which is independent of $t$, we define

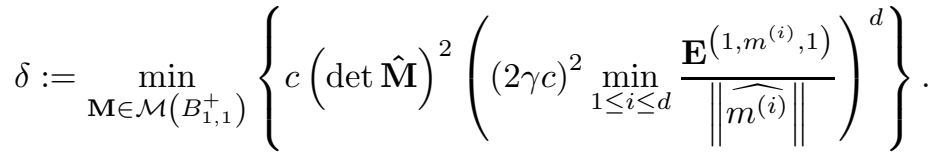

Obviously $\delta>0$, leading to

$$
\operatorname{det} \sigma(t) \geq \delta\|t\|^{2 d}
$$

for sufficiently small $\|t\|$. The estimate from above is derived in a similar fashion.

Remark 4.19. The algorithms for verifying the rigidity of the lattice are based on the representation of the determinant of the symbol as in Lemma 4.8. The restriction to the case $q=1$ for vector potentials is related to the fact that this representation is available only for $q=1$.

4.3. The inverse of the symbol $(q=1$ and general $d)$. We have proven that the symbol has the representation

$$
\sigma(t)=4 \sum_{n \in B_{1,1}^{+}} \mathbf{E}^{(1, n, 1)} \sin ^{2} \frac{\langle n, t\rangle}{2} .
$$

The estimate of the inverse symbol is obtained by Cramer's rule.

Theorem 4.20. Let $q=1$ and let condition (4.2) be satisfied. Then, the coefficients of the inverse symbol satisfy

$$
\left|\left(\sigma^{-1}\right)_{i, j}(t)\right| \leq C\|t\|^{-2} \quad \forall t \in[-\pi, \pi]^{d} \backslash\{0\} .
$$

Proof. Every coefficient of the symbol matrix $\sigma(t)$ can be estimated by

$$
\left|\sigma_{i, j}(t)\right| \leq C\|t\|^{2} .
$$

The definition of the adjugate matrix $\sigma^{(i, j)}$ implies

$$
\left|\operatorname{det} \sigma^{(i, j)}(t)\right| \leq C\|t\|^{2 d-2} .
$$

Cramer's rule in combination with Lemma 4.18 yields

$$
\left|\left(\sigma^{-1}\right)_{i, j}(t)\right| \leq C\|t\|^{-2} \text {. }
$$




\section{ACKNOWLEDGMENTS}

This work was supported by the Army Research Office Grant DAAD19-99-0149, by the National Science Foundation Grant DMS-9802367, by the Swiss Federal Office for Education and Science Grant 01.0025-1/2 (as a part of the HMS 2000Research Training Network "Homogenization and Multiple Scales" of the European Union), and by the Swiss National Science Foundation Grant 21-58891.99.

\section{REFERENCES}

[1] L. Asimov and B. Roth. The Rigidity of Graphs. Trans. Am. Math. Soc., 245:279-289, 1978. MR 80i:57004a

[2] I. Babuška and S. A. Sauter. Efficient Solution of Lattice Equations by the Recovery Method. Part 1: Scalar Elliptic Problems. TICAM REPORT 02-39, University of Texas at Austin, 2002.

[3] W. Brown. Matrices over Commutative Rings. Marcel Dekker, New York, 1992. MR 93k:15028

[4] G. Constantinides and A. C. Payatakes. A Three Dimensional Network Model for Consolidated Porous Media. Basic Studies. Chem. Eng. Comm., 81:55-81, 1980.

[5] H. Crapo and W. Whiteley. Encyclopedia of Mathematics and Its Applications, chapter "The Geometry of Rigid Structures". Cambridge Univ. Press, 1989.

[6] V. Deshpande, N. Fleck, and M. Ashby. Effective properties of the octet truss material. Technical report, Cambridge Univ., Dept. Eng., 2000.

[7] I. Fatt. The Network Model of Porous Media. Trans. Am. Inst. Mem. Metall Pet. Eng., 207:144-181, 1956.

[8] L. Gibson and M. Ashby. Cellular Solids, Structures and Properties. Pergamon Press, Exeter, 1989.

[9] M. L. Glasser and J. Boersma. Exact Values for the Cubic Lattice Green Functions. J. Phys., 133:5017-5023, 2000. MR 2001i:82022

[10] J. C. Hansen, S. Chien, R. Skalog, and A. Hoger. A Classic Network Model Based on the Structure of the Red Blood Cell Membrane. Biophy. J., 70:146-166, 1996.

[11] G. S. Joyce. On the Simple Cubic Lattice Green Functions. Proc. Roy. Soc., London Ser. A, 445:463-477, 1994. MR 95j:33041

[12] G. Laman. On Graphs and Rigidity of Plane Skeletal Structures. Journal of Engineering Math., 4:331-340, 1970. MR 42:4430

[13] A. Maradudin, E. Montroll, G. Weiss, R. Herman, and H. Milnes. Green's functions for monoatomic simple cubic lattices. Acad. Roy. Belg. Cl. Sci. Mem.Coll. in-4deg, 14(2):176, 1960. MR 22:7440

[14] P. G. Martinson. Fast Multiscale Methods for Lattice Equations. PhD thesis, University of Texas at Austin, 2002.

[15] A. Noor. Continuum modelling for repetitive lattice structures. Appl. Mech. Rev., 41(7):285296, 1988.

[16] M. Ostoja-Starzewski. Lattice Models in Micromechanics. App. Mech. Review, 55(1):35-60, 2002.

[17] M. Ostoja-Starzewski, P. Y. Shang, and K. Alzebdoh. Spring Network Models in Elasticity and Fractures of Composites and Polycristals. Comp. Math. Sci., 7:89-93, 1996.

[18] G. I. Pshenichnov. Theory of Lattice Plates and Shells. World Scientific, Singapore, 1993. MR 97i:73001

[19] R. Zurmühl and S. Falk. Matrizen und Ihre Anwendungen. Springer Verlag, Berlin, Heidelberg, 5 edition, 1984. MR 86a:15002

University of Texas at Austin, ICES, 1 University Station, C0200, Austin, Texas $78712-0027$

E-mail address: babuska@ticam.utexas.edu

University of Zurich, Institute of Mathematics, Winterhurestr. 190, CH 8057 Zurich, SWITZERLAND

E-mail address: stas@math.unizh.ch 